\title{
Effectiveness of forward obstacles collision warning system based on deceleration for collision avoidance
}

\section{$\operatorname{AUTHOR}(\mathrm{S})$ :}

Takada, Shota; Hiraoka, Toshihiro; Kawakami, Hiroshi

\section{CITATION:}

Takada, Shota ... [et al]. Effectiveness of forward obstacles collision warning system based on deceleration for collision avoidance. IET Intelligent Transport Systems 2014, 8: 570-579

\section{ISSUE DATE:}

2014-09

URL:

http://hdl.handle.net/2433/209743

\section{RIGHT:}

This paper is a postprint of a paper submitted to IET Intelligent Transport Systems and is subject to Institution of Engineering and Technology Copyright. If accepted, the copy of record will be available at IET Digital Library:; (c) The Institution of Engineering and Technology 2013; この論文は出版社版でありません。引用の際には出版社版をご確認ご 利用ください。; This is not the published version. Please cite only the published version. 
The Effectiveness of Forward Obstacles Collision Warning System Based on Deceleration for Collision Avoidance

\begin{tabular}{|r|l|}
\hline Journal: & IET Intelligent Transport Systems \\
\hline Manuscript ID: & ITS-2013-0024.R2 \\
\hline Manuscript Type: & Research Paper \\
\hline Date Submitted by the Author: & 11 -Oct-2013 \\
\hline Complete List of Authors: & $\begin{array}{l}\text { Takada, Shota; Kyoto University, Graduate School of Informatics } \\
\text { Hiraoka, Toshihiro; Kyoto University, Graduate School of Informatics } \\
\text { Kawakami, Hiroshi; Kyoto University, Graduate School of Informatics }\end{array}$ \\
\hline Keyword: & $\begin{array}{l}\text { COLLISION WARNING, HUMAN FACTORS, HUMAN MACHINE INTERACTION, } \\
\text { SAFETY }\end{array}$ \\
\hline \multicolumn{2}{|c}{} \\
\hline
\end{tabular}

SCHOLARONE ${ }^{m}$

Manuscripts 


\title{
The Effectiveness of Forward Obstacles Collision Warning System Based on Deceleration for Collision Avoidance
}

\author{
Shota TAKADA ${ }^{1^{*}}$, Toshihiro HIRAOKA ${ }^{2^{*}}$, Hiroshi KAWAKAMI ${ }^{3^{*}}$ \\ 1. Master Course Student, 2. Assistant Professor, 3. Associate Professor \\ Graduate School of Informatics, Kyoto University \\ Yoshida Honmachi, Sakyo-ku, Kyoto, 606-8501, Japan \\ TEL\&FAX: +81-75-753-5042 \\ E-mail: \{takada, hiraoka, kawakami\}@sys.i.kyoto-u.ac.jp
}

\begin{abstract}
In our previous study, we proposed Deceleration for Collision Avoidance (DCA) as an index to evaluate collision risks against forward obstacles and examined the effectiveness of our Forward Obstacles Collision Warning System (FOCWS) based on DCA. In the present manuscript, we improve the visual interface of the FOCWS, and conduct driving simulator experiments to quantitatively evaluate the effectiveness of the improved FOCWS in situations where a preceding vehicle decelerates abruptly. The experimental results revealed that the FOCWS based on DCA was effective in assisting drivers to shorten the reaction time and to avoid collisions. Moreover, in the subjective assessment questionnaire, a significant number


of experimental participants reported that the FOCWS based on DCA could evaluate collision risks more properly compared to the FOCWS based on a time-to-collision (TTC). 


\section{Introduction}

Various countermeasures have been proposed by the Japanese Government's Eighth Fundamental Traffic Safety Program ${ }^{[1]}$ to reduce traffic accidents, of which, Advanced Safety Vehicles (ASVs) are the most well-known examples. ASV is defined as an intelligent vehicle capable of utilizing information-communication technology. Our research focuses on the Forward Obstacles Collision Warning System (FOCWS), which is a warning function of a forward collision damage mitigation braking system, and is one of the practical technologies employed in ASVs. Recently, there are various kinds of Collision Avoidance Systems (CASs) which equip the FOCWS function and an automatic braking function. They can detect obstacles by using stereo camera, laser radar sensor, or milliwave radar sensor ${ }^{[2,3,4,5]}$. Many researches have examined effects of the systems on drivers' behavior, by using driving simulator or actual vehicle ${ }^{[6,7,8,9,10]}$.

Our previous study proposed Deceleration for Collision Avoidance (DCA) as an index for use when evaluating collision risks against forward obstacles ${ }^{[11]}$, and applied it as a provisional warning threshold for the FOCWS. Additionally, driving simulator experiments were performed to evaluate the effectiveness of FOCWS based on DCA ${ }^{[12]}$. However, the results did not sufficiently clarify the quantitative effectiveness of FOCWS, nor did the study examine negative aspects of FOCWS, because they did not compare the case of driving with 
and without the FOCWS.

Moreover, in the first paper ${ }^{[11]}$ which proposed DCA, the calculation process of DCA assumed that a following vehicle initiates a uniform motion within a driver's constant reaction time, and then, the assumption causes underestimation and overestimation of the collision risk when the following vehicle accelerates and decelerates, respectively. Therefore, in our subsequent efforts, we improved the DCA calculation process and performed numerical simulations ${ }^{[13]}$. The improved DCA is based on an expanded assumption that the following vehicle initiates a uniformly-accelerated motion within the reaction time. The results indicated that, when compared to conventional DCA, the improved DCA could evaluate collision risks more effectively.

Accordingly, in the present study, driving simulator experiments were performed to evaluate the quantitative effectiveness of the FOCWS based on the improved DCA in situations involving abrupt deceleration of a preceding vehicle, and to clarify driving behavior variations.

\section{Deceleration for Collision Avoidance}

\subsection{DCA Outline}


DCA $\left[\mathrm{m} / \mathrm{s}^{2}\right]$ is a measurement that indicates the minimum required deceleration of a following vehicle necessary to avoid a collision with a forward obstacle, such as a preceding vehicle ${ }^{[11,}$ 13]. DCA, in a situation where a preceding vehicle maintains its current acceleration, is defined as overt DCA (ODCA), and the ongoing DCA that is based on the assumption that the preceding vehicle will decelerate abruptly at any moment is defined as potential DCA (PDCA).

\subsection{Principle symbols}

Figure 1 shows the variables used in the present paper. This paper adopts the same variables defined in the previous study ${ }^{[14]}$. It assumes that the preceding vehicle initiates motion and accelerates in a uniform manner, with acceleration $a_{p 0}$, and that the following vehicle initiates motion and accelerates in a uniform manner, with acceleration $a_{f 0}$ within a driver's reaction time $T$, and with acceleration $a_{f T}$ after the reaction time $T$. Note that the relative position $x_{r}(=$ $x_{f}-x_{p}$ ) becomes negative when the following vehicle is behind the preceding vehicle. Therefore, the inter-vehicular distance for the following state can be calculated by reversing the sign of the relative distance $x_{r}$.

\subsection{Driver reaction time}

The DCA calculation process requires knowledge of the reaction time $T$ of the following 
vehicle driver. Note that the "reaction" in this context refers to the process that begins the moment the driver decides to act and extends to the moment the action is complete. As mentioned above, DCA is defined with the assumption that the following vehicle continues accelerating during the reaction time $T$. This indicates that the driver will be unable to avoid a collision in situations where the impact will occur during the reaction time - even if warned by the FOCWS. The condition for collision avoidance within the reaction time becomes as defined in the equation below:

$$
x_{r 0}+v_{r 0} T+\frac{1}{2} a_{r 0} T^{2}<0
$$

Here, the driver's reaction time $T$ is defined by

$$
T= \begin{cases}1.2[\mathrm{~s}] & \left(b_{r}=0\right) \\ 0.2[\mathrm{~s}] & \left(b_{r}>0\right)\end{cases}
$$

where $b_{r}$ represents the degree of brake pedal depression $\left(0 \leq b_{r} \leq 1\right)^{[13]}$. In our previous paper

[12], driver reaction time $T$ was defined as a constant value: 1.21 [s], because the simulator experiments revealed that 90 percent of all recorded reaction times were within that value. However, reaction times will be significantly less than the abovementioned value in situations where the driver is already depressing the brake pedal. Therefore, in cases when the driver is already depressing the brake pedal, the reaction time is shortened to 0.2 [s]

\subsection{Overt DCA (ODCA)}


ODCA, $\alpha_{o}\left[\mathrm{~m} / \mathrm{s}^{2}\right]$, is defined as the DCA occurring when the preceding vehicle maintains its current acceleration.

$$
\alpha_{o}= \begin{cases}0 & \left(a_{p 0} \geq 0, v_{p 0}+a_{p 0} T \geq v_{f 0}+a_{f 0} T \text { or } a_{p 0}<0,-\frac{v_{p 0}}{a_{p 0}}<t_{1}, v_{f 0}+a_{f 0} T \leq 0\right) \\ -a_{f 1} & \left(a_{p 0} \geq 0, v_{p 0}+a_{p 0} T<v_{f 0}+a_{f 0} T \text { or } a_{p 0}<0, T<t_{1}<-\frac{v_{p 0}}{a_{p 0}}\right) \\ -a_{f 2}\left(a_{p 0}<0,-\frac{v_{p 0}}{a_{p 0}}<t_{1}, v_{f 0}+a_{f 0} T>0\right)\end{cases}
$$

where $a_{f 1}, a_{f 2}, t_{1}$ are defined as follows:

$$
\begin{aligned}
& a_{f 1}=\frac{v_{r 0}^{2}+2 a_{p 0} x_{r 0}+\left(2 v_{r 0}+a_{r 0} T\right) a_{f 0} T}{x_{r 0}+2 v_{r 0} T+a_{r 0} T^{2}} \\
& a_{f 2}=\frac{\left(v_{f 0}+a_{f 0} T\right)^{2}}{2 x_{r 0}+2 v_{f 0} T+a_{f 0} T^{2}+\frac{v_{p 0}^{2}}{a_{p 0}}} \\
& t_{1}=-\frac{2 x_{r 0}+v_{r 0} T}{a_{r 0} T+v_{r 0}} \leq-\frac{v_{p 0}}{a_{p 0}} .
\end{aligned}
$$

\subsection{Potential DCA (PDCA)}

PDCA, $\alpha_{p}\left[\mathrm{~m} / \mathrm{s}^{2}\right]$, is defined based on the assumption that the preceding vehicle suddenly decelerates at a constant rate of $0.6[\mathrm{G}]\left(=5.88\left[\mathrm{~m} / \mathrm{s}^{2}\right]\right)$. The PDCA calculation process is basically the same as that for the ODCA. The difference is that the preceding vehicle's acceleration $a_{p 0}$ is set at $-5.88\left[\mathrm{~m} / \mathrm{s}^{2}\right]$ when it is moving forward $\left(v_{p 0}>0\right)$, or set at $0\left[\mathrm{~m} / \mathrm{s}^{2}\right]$ when the vehicle stops $\left(v_{p 0}=0\right)$. 
Case 1: the preceding vehicle is moving forward

The PDCA in situations when the preceding vehicle is moving forward can be expressed by

$$
\alpha_{p}= \begin{cases}0 & \left(v_{p 0}>0, \frac{v_{p 0}}{5.88}<t^{\prime}{ }_{1}, v_{f 0}+a_{f 0} T \leq 0\right) \\ -a_{f 1}^{\prime}\left(v_{p 0}>0, T<t^{\prime}{ }_{1} \leq \frac{v_{p 0}}{5.88}\right) & \left(v_{p 0}>0, \frac{v_{p 0}}{5.88}<t^{\prime}{ }_{1}, v_{f 0}+a_{f 0} T>0\right) \\ -a_{f 2}^{\prime}\left({ }^{\prime}\left(v^{\prime}\right)\right. & \end{cases}
$$

where $a_{f 1}^{\prime}, a_{f 2}^{\prime}$, and $t_{1}^{\prime}$ are obtained from Eqs. (4), (5), and (6), respectively, by substituting $-5.88\left[\mathrm{~m} / \mathrm{s}^{2}\right]$ for $a_{p 0}$.

Case 2: the preceding vehicle stops

The PDCA in cases when the preceding vehicle stops can be expressed by

$$
\alpha_{p}= \begin{cases}0 & \left(v_{p 0}=0, a_{p 0}=0, v_{f 0}+a_{f 0} T \leq 0\right) \\ -a^{\prime \prime}{ }_{f 1} & \left(v_{p 0}=0, a_{p 0}=0, v_{f 0}+a_{f 0} T>0\right)\end{cases}
$$

where $a^{\prime \prime}{ }_{11}$ describes $a_{f 1}$ when zero is substituted into $v_{p 0}$ and $a_{p 0}$. For additional details on the

DCA derivation process, please refer to our previous study ${ }^{[13]}$.

\section{Driving simulator experiments}

\subsection{Previous DCA-FOCWS}


The experimental participants, in our previous driving simulator experiments ${ }^{[12]}$, utilized a FOCWS based on the conventional DCA. It provides visual information as shown in Fig. 2 (a), and a collision warning. The visual interface consists of an inner yellow bar that represents the PDCA value $\alpha_{p}$ and an outer red bar that represents the ODCA value $\alpha_{o}$. The bars expand and contract vertically, and the top and bottom of the bars indicate 6.0 and 0 $\left[\mathrm{m} / \mathrm{s}^{2}\right]$ respectively. The color of bars and the extension direction are designed to satisfy S-R compatibility ${ }^{[15]}$. The collision warning is sounded while the red bar (ODCA) exceeds the threshold $\left(=4.0\left[\mathrm{~m} / \mathrm{s}^{2}\right]\right)$, which was determined in our previous experiments ${ }^{[12]}$.

\subsection{Proposed DCA-FOCWS}

The previous experiments ${ }^{[12]}$ also suggested that the visual interface might cause drivers' confusion because of its complexity. For example, one of the experimental participants answered to the questionnaire about the interface; "I did not understand whether I should watch out for the red or the yellow bar." Therefore, for this study, the interface was modified as shown in Fig. 2 (b). The center bar represents the ODCA value $\alpha_{o}$, while the top and bottom indicate 6.0 and $0\left[\mathrm{~m} / \mathrm{s}^{2}\right]$ respectively. The bar color is displayed in light blue when the deceleration is sufficient to avoid collision $\left(-a_{f 0} \geq \alpha_{o}\right)$ and changes to orange when deceleration is insufficient to avoid collision $\left(-a_{f 0}<\alpha_{o}\right)$ based on the following vehicle's current deceleration $a_{f 0}$. As for the PDCA, a yellow frame appears around the center bar in situations 
where the PDCA exceeds $4.0\left[\mathrm{~m} / \mathrm{s}^{2}\right]$. Furthermore, the FOCWS provides an audible collision warning when the ODCA exceeds $4.0\left[\mathrm{~m} / \mathrm{s}^{2}\right]$ as same as the previous DCA-FOCWS ${ }^{[12]}$, and it continues unless $\alpha_{o}$ falls below $2.0\left[\mathrm{~m} / \mathrm{s}^{2}\right]$ in order not to provide a very short-term warning.

\subsection{FOCWS based on TTC (TTC-FOCWS)}

TTC is one of the most well-known indices to evaluate the collision risk, and it is employed as an index to determine the timing of collision warning provision in many FOCWSs. Consequently, in the present study, the simulator experiments were prepared utilizing a FOCWS based on the TTC $\left(=-x_{r} / v_{r}\right)$, called a TTC-FOCWS (Fig. 2 (c)), which was adopted to be compared to the proposed DCA-FOCWS. The TTC value is represented by the orange bar. The top and bottom denote a range from 0 to 12 [s] in order to align the elastic direction of the bar and the threshold-line of collision warning to those of the DCA-FOCWS. A collision warning is provided while the TTC is less than $4.0[\mathrm{~s}]$, which was found to be a proper threshold for a collision warning timing of the same driving simulator environment in our previous study ${ }^{[16]}$.

\subsection{Experimental conditions}

Figure 3 shows a front view of the driving simulator, which was same with our previous studies ${ }^{[12,14]}$. It is a fixed-base handmade driving simulator which equips a digital signal 
processor (DSP) unit and interfaces such as a steering wheel, a gas pedal, a brake pedal, and a meter display. CarSim RT-Linux, which is installed on the DSP unit, calculates 19 degree-of-freedom vehicle dynamics in real time based on the drivers' inputs. The experimental participants were twelve males and four females, ranging in age from 20 to 36 (Ave. 26.1 yrs). Each participant drove a virtual vehicle along a course consisting of a two-lane, one-way straight road, the width and length of which were $7[\mathrm{~m}]$ and $6[\mathrm{~km}]$, respectively. The following vehicle, which an experimental participant drove, was set to travel in the left lane along with two vehicles that traveled in the same direction. A preceding vehicle traveled in front of the following vehicle and a parallel-running vehicle traveled in the right lane.

Each experimental participant drove the course three times for each of three driving conditions. During each simulator run, the preceding vehicle executed different dangerous deceleration patterns, as shown in Fig. 4, twice per each condition, in random order.

We determined eight evaluation sections that included one dangerous deceleration pattern. In order to prevent experimental participants from anticipating pending events, the preceding vehicle randomly accelerated or decelerated within the range from $0.1[\mathrm{G}]$ to 0.3 [G] outside the evaluation sections. The maximum velocity of the preceding vehicle was $80[\mathrm{~km} / \mathrm{h}]$, and 
the preceding vehicle stopped 18 times during the course for each driving condition. The velocity of the parallel-running vehicle traveling in the right lane at time $t$ was set to the preceding vehicle's velocity at time $t-1[\mathrm{~s}]$.

\subsection{Experimental participant instructions}

The experimental participants were given the following instructions:

1) Drive in the left lane and follow the preceding vehicle.

2) Take proper avoidance behavior when you detect danger.

3) Follow the preceding vehicle within a distance sufficient to prevent the parallel-running vehicle from cutting in ahead.

4) Engage in the mental arithmetic task as long as it does not disturb driving ability.

For number 3), the right edge of the meter display was set to turn yellow when the following vehicle was too far from the preceding vehicle, and we added the instruction: "Catch up with the preceding vehicle when indicated by the display. " For number 4), a mental arithmetic task was set as a sub-task that required experimental participants to listen to two numbers between $1 \sim 9$ spoken every two seconds, add them together, and then provide verbal answers. The purpose of the sub-task was to reproduce the driver workload equivalent to the real driving situation. 


\subsection{Experimental procedure}

Before the experiments began, each experimental participant performed practice driving to familiarize themselves with the driving simulator environment. Then they drove the course under three types of driving conditions, as follows:

a) w/o-condition: driving without the FOCWS

b) TTC-condition: driving with the TTC-FOCWS

c) DCA-condition: driving with the DCA-FOCWS

To reduce order effects, the experimental participants were divided into four groups, and the experimental order was varied for each.

Prior to the TTC- and DCA-conditions, the experimental participants were provided an explanation of the system, after which they performed practice driving to familiarize themselves with the FOCWS. Note that the explanation provided by the experimenter regarding the FOCWS behavior was limited to the following: "The bar extends upward as the possibility of collision increases, and a collision warning is provided when the risk exceeds the red line." In other words, they were not provided background explanations about TTC, DCA, or even the best way to use the FOCWS. During the practice driving sessions, they familiarized themselves with the behavior of the visual interface and collision warning by 
viewing an auto-running demonstration. Each experimental participant was also given several opportunities to use the FOCWS when driving the simulated vehicle unsupervised.

\section{Experimental results and discussion}

\subsection{Results of collision avoidance}

Figure 5 (a) shows the average number of collisions, in all evaluation sections, for each driving condition. Note that the total number of abrupt decelerations simulated rear-end collision was eight for each driving condition. The one-way analysis of variance (ANOVA) revealed that a main effect of the driving condition was significant $(F(2,30)=4.33, p<.05)$, and Shaffer's multiple comparison test clarified that the number of DCA-condition collisions (at 5\%) was statistically smaller than that of w/o- and TTC-conditions. The results indicate that the DCA-FOCWS is effective at reducing collisions.

Figures 5 (b) and (c) show the average brake-on TTC and brake-on ODCA, respectively. The brake-on TTC (ODCA) represents the value of TTC (ODCA) at the moment when the following vehicle's driver depressed the brake pedal in reaction to the preceding vehicle's abrupt deceleration. One-way ANOVA revealed that the main effect of the driving condition on each index was significant $(F(2,30)=12.91, p<.001$ and $F(2,30)=11.50, p<.001)$. Multiple comparison tests clarified that the brake-on TTC of the DCA-condition was 
statistically larger than those of other two conditions (at 5\%), and that the brake-on ODCA level of the DCA-condition was statistically smaller than those of other two conditions (at 5\%). This indicates that provision of the DCA-FOCWS contributes to shortening driver reaction times, and therefore, the TTC and ODCA at the moment when drivers take avoidance action becomes safer.

Figures 6 (a) and (b) show the number of collisions and the brake-on TTC for each deceleration pattern of the preceding vehicle. Two-way ANOVA revealed that the interaction between the driving condition and the deceleration pattern was significant in the number of collisions $(F(6,90)=3.45, p<.01)$ and in the brake-on TTC $(F(6,90)=3.84, p<.01)$. Furthermore, the main effect of the driving condition was significant for Pattern 1 in the former $(F(2,30)=11.67, p<.001)$, and for Patterns 1 and 3 in the latter $(F(2,30)=12.08, p$ $<.001$ and $F(2,30)=6.20, p<.01)$. According to the multiple comparison tests, all those of the DCA-condition were statistically small or large (at 5\%) compared to the w/o-condition and TTC-condition.

These results suggest that the DCA-FOCWS was very effective at enhancing collision avoidance, especially in Pattern 1. In Pattern 1, the following vehicle would accelerate to follow the preceding vehicle while it was accelerating and the preceding vehicle would 
abruptly decelerate just after it reached $80[\mathrm{~km} / \mathrm{h}]$. Because the improved DCA ${ }^{[13]}$ reflects the current following vehicle's acceleration, the collision warning can be provided earlier in situations where the following vehicle is accelerating. This indicates that the collision warning was provided immediately in reaction to the preceding vehicle's abrupt deceleration, and thus contributed to reducing collisions by shortening the following vehicle's driver reaction time.

\subsection{Reaction time for preceding vehicle and collision warning}

Figure 7 (a) illustrates the average reaction time $R_{t 1}$ to the preceding vehicle's deceleration for each driving condition. $R_{t 1}$ is defined as the time interval between the preceding vehicle's deceleration and the moment when the following vehicle's driver depressed the brake pedal. $R_{t 1}$ in the DCA-condition was slightly smaller compared to other two conditions while there was no significant difference.

Figure 7 (b) shows the reaction time $R_{t 2}$ for each deceleration pattern of the preceding vehicle in the TTC-condition and DCA-condition. $R_{t 2}$ is defined as the reaction time from the moment FOCWS provided the collision warning to the moment following vehicle's driver depressed the brake pedal. $R_{t 2}$ in the DCA-condition was obviously larger than the TTC-condition. It suggests that the DCA-FOCWS provided collision warning before the experimental participants intended to put on the brake. On the other hand, $R_{t 2}$ of Pattern 1 in the 
TTC-condition was negative value. It means that many participants depressed the brake pedal before the TTC-FOCWS provided warning. Similarly, in the cases of Patterns $2 \sim 4$, some participants would intend to put on the brake before the collision warning.

\subsection{Effect of DCA-FOCWS on driving behavior}

Four experimental participants (Subs. \#3, \#8, \#11, \#15) exhibited similar distinctive driving behaviors. Table 1 (a) describes the total amount of time where PDCA exceeded $4.0\left[\mathrm{~m} / \mathrm{s}^{2}\right]$ in all evaluation sections for the four participants. The results show that the times decreased significantly in the DCA-condition. This trend was only observed in the four participants because the average time of all experimental participants in the w/o-, TTC- and DCA-conditions were 207.8, 227.9, and 204.5 [s], respectively. Furthermore, Table 1 (b) shows that the number of collisions for the four participants also decreased in the DCA-condition.

As mentioned above, a yellow frame is displayed by the DCA-FOCWS in situations where PDCA $>4.0\left[\mathrm{~m} / \mathrm{s}^{2}\right]$. The experimental results indicate that the four participants appeared to have controlled the following vehicle velocity in a manner that prevented the frame from appearing (not exceeding $4.0\left[\mathrm{~m} / \mathrm{s}^{2}\right]$ in PDCA), even though no experimental participants were provided instructions on how to use the FOCWS. 
Figure 8, for example, shows the time series data of Sub. \#8 engaged in driving Pattern 4 of the DCA-condition. In that pattern, the preceding vehicle traveled at $50[\mathrm{~km} / \mathrm{h}]$ for more than 60 seconds before it decelerated at a rate of $0.4[G]$. The figure illustrates that Sub. \#8 would have controlled velocity so as not to turn on the frame.

Consequently, the results indicate that the four participants were able to reduce the number of collisions because they maintained a safer inter-vehicular distance by adjusting vehicle velocity in order to prevent the yellow frame from appearing.

Figure 9 (a) illustrates the combined number of collisions for the other 12 participants (excluding the four abovementioned participants). The number of collisions for the DCA-condition (1.75 times in average, Figure 9 (a)) was larger than that recorded for the four abovementioned participants $(0.25$ times in average, Table $1(\mathrm{~b}))$, but there was no statistical difference between them. Moreover, as shown in Figs. 9 (b) and (c), the brake-on TTC and the brake-on ODCA of these twelve participants have similar tendencies of those in the case of all participants (Figs.5 (b) and (c)).

\section{Subjective evaluation}




\subsection{Contents of the questionnaire}

This section discusses results of the pencil-and-paper questionnaire for subjective evaluation.

A questionnaire Q.1, which was performed after TTC/DCA conditions, consisted of the following three questions 1-a $\sim 1$-c. Questions 1-a and 1-b were 5-grade scale questions, and Question 1-c was a free-answer question.

1-a) Was the provided information useful?

1-b) Could you take proper driving behavior according to the provided information?

1-c) For question 1-b, how did you behave?

Experimental participants answered Questions 1-a and 1-b repeatedly with respect to three types of information; 1) the collision warning, 2) the bar of the visual interface, and 3) the frame of the visual interface (DCA condition).

A questionnaire Q.2, which was conducted at the end of experiment, consisted of Questions 2-a $\sim 2$-e on a 5-grade scale in order to explore comparisons and subjective evaluations between the TTC-FOCWS and DCA-FOCWS. For Question 2-e-2, experimental participants were asked to provide a written response. The questions were as follows: 
2-a) Did you find differences between the systems (TTC-FOCWS and DCA-FOCWS)?

2-b) Which system is easier to understand with respect to the contents of visual interface?

2-c) Which system do you think is more effective in terms of collision risk evaluation?

2-d) Which system do you think is more effective in terms of collision warning timing?

2-e) Which system would you want to use in an actual driving environment?

2-e-2) For Question 2-e, what is the reason you chose the answer?

For Questions 2-b 2-e, the experimental participants were asked to provide their answers in the following range: -2 : TTC-FOCWS $\sim 0$ : neither $\sim 2$ : DCA-FOCWS.

Moreover, experimental participants answered the driving style questionnaire (DSQ) ${ }^{[17]}$.

\subsection{Evaluation for the FOCWS}

\subsubsection{About the usefulness of the FOCWS}

Figure 10 (a) shows the average scores for Questions 1-a and 1-b. As for Question 1-a, the score for collision warning of the DCA-FOCWS was statistically larger than that of the TTC-FOCWS. The result indicates that a lot of participants thought the collision warning of the DCA-FOCWS was useful, and it is consistent with the quantitative results, mentioned in section 4.2 , that the reaction time $R_{t 2}$ became larger in the DCA-condition compared to the 
TTC-condition.

For Question 2-a, 12 of 16 experimental participants answered that they felt a difference between the two systems. Seven participants answered "I felt a difference" and five participants answered "I felt a small difference". Figure 10 (b) shows the range of scores for Questions 2-b 2-e. It shows that for Questions 2-b and 2-e, the answer was split down the middle, which indicates that opinions differed from participant to participant in terms of the visual interface understandability or whether they would like to use the system in an actual driving environment. On the other hand, the overall score of 2 for Questions 2-c and 2-d is somewhat remarkable because they indicate that a significant number of experimental participants believed the DCA-FOCWS to be superior in terms of the collision risk evaluation.

\subsubsection{Correlations between subjective evaluation and DSQ score}

Here, the experimental participants were classified into the following three groups based on the results of Question 1-a.

Group 1: Subs. \#1, \#2, \#8, \#12, and \#14

$\rightarrow$ Participants who answered that the DCA-FOCWS's bar was useful (score: 4 or 5) while 
the frame was not useful (score: $1 \sim 3$ ).

Group 2: Subs. \#3, \#5, and \#11

$\rightarrow$ Participants who answered that the DCA-FOCWS's frame was useful (score 4 or 5) while the bar was not useful (score $1 \sim 3$ ).

Group 3: Subs. \#4, \#6, \#7, \#9, \#10,\#13, \#15, and \#16

$\rightarrow$ Other participants.

Figure 11 shows the average scores of DSQ for each group. In Group 2, the score of "Hesitation for drive" and "Anxiety about accident" were higher compared to other two groups. Consequently, it implies that the drivers who have a negative attitude to driving tend to prefer the PDCA frame compared to the ODCA bar because they try to perform more careful driving. Furthermore, it can be derived from their driving behaviors mentioned in section 4.3 that Subs. \#3 and \#11 of Group 2 maintained a safer inter-vehicular distance in order to prevent the PDCA frame from appearing.

\subsection{Free description about the comparison of the FOCWS}

For Question 1-b as shown in Fig. 10 (a), majority of the experimental participants reported that they could take proper behavior according to the collision warning or visual information of the FOCWS. Some participants answered Question 1-c of DCA-condition as follows: 
Sub. \#4, \#9, and \#14: "I tried to keep the bar color blue."

Sub. \#3, \#5, \#11, and \#15: "I tried to open the inter-vehicular distance to the preceding vehicle when the frame appeared."

These answers indicate that they drove their vehicles according to their own criteria based on the information of the DCA-FOCWS.

Moreover, in TTC-condition, some participants answered as follows:

Sub. \#4: "I did not use the system, and decelerated the car by my feeling of distance."

Sub. \#15: "The FOCWS provided the collision warning very frequently. I pressed the brake pedal every time it was provided."

Accordingly, these participants might think that the information provided by the TTC-FOCWS was not appropriate, and therefore they could not find their own criteria how to utilize the information of the TTC-FOCWS.

Next, we will consider the Question 2-e-2 answers of those experimental participants that 
chose the TTC-FOCWS (score -1 or -2) in Question 2-e:

Sub. \#5, \#7, and \#10: "The visual interface was simple and understandable."

Sub. \#13: "I couldn't take the time to determine the color of the bar (in the case of the DCA-FOCWS) when driving."

These answers indicated the reason why the participants chose the TTC-FOCWS in Question 2-e might be its simplicity. Furthermore, no opinions were stated that indicate the participants thought the TTC-FOCWS was superior in terms of the collision risk evaluation.

On the other hand, the experimental participants who chose the DCA-FOCWS (score 1 or 2)

in Question 2-e answered in Question 2-e-2 as follows:

Sub. \#1: "Collisions did not occur when using the DCA-FOCWS. Furthermore, the timing of the collision warning was also appropriate."

Sub. \#4: "Using the system, I was able to determine whether the situation was safe or not and it was useful for improving my driving skill."

Sub. \#9: "Driving became easy when using the system. The changing color of the bar was also understandable." 
Sub. \#15: "Driving became more dangerous when using the TTC-FOCWS."

These descriptions indicated that they chose DCA-FOCWS in Question 2-e because they thought the collision risk evaluation provided by DCA-FOCWS was more appropriate. In particular, Sub. \#4 underscored the effectiveness of the system when he answered, "The system was useful for improving my driving skill."

\subsection{Correlations between subjective evaluation and collision avoidance performance}

Experimental participants were classified into the following two groups based on subjective evaluations of the results for Question 2-e.

Group A: Subs. \#1,\#3,\#4,\#6, \#8,\#9,\#11\#15 $\rightarrow$ participants who chose the DCA-FOCWS

Group B: Subs. \#2, \#5,\#7,\#10,\#12,\#13,\#14,\#16 $\rightarrow$ the others

Figure 12 shows the results of collision avoidance performance (number of collisions, brake-on TTC, and brake-on ODCA) for each group. In Group A, the main effects of the driving condition were significant in all three indices, and all three became safer in the DCA-condition. For Group B, there was no statistically-significant difference between indices. 
These results strengthen the implication that Group A participants chose the DCA-FOCWS due to their satisfaction with its collision risk evaluations. Those participants improved their collision avoidance performance by using the DCA-FOCWS and expressed desires to use it in actual driving environments. Conversely, for Group B, collision avoidance performance did not improve as significantly under the DCA-condition when compared to the other conditions. Thus, it is considered likely that the participants of Group B would not be interested in using the DCA-FOCWS in actual driving environments because it would not improve their collision avoidance performance.

\section{Conclusions}

In this paper, we reported the results of experiments performed using a driving simulator to evaluate the quantitative effect of the FOCWS, based on our improved DCA (DCA-FOCWS), in situations where the preceding vehicle decelerates abruptly. The experimental results showed that the DCA-FOCWS could be effective in assisting drivers to avoid collisions. In answers to the subjective assessment questions, a significant number of experimental participants said the DCA-FOCWS properly evaluated collision risks, and that the timings of the collision warnings were appropriate.

Some participants answered that the contents of the visual information was not 
understandable. As a future work, therefore, we have to discuss other presentation methods of the DCA in order to reduce drivers' visual workload, e.g., a Head-Up Display ${ }^{[18,19]}$ and a haptic feed-back system.

The present study performed the driving simulator experiments on the assumption that the FOCWS does not provide false alarms and missing alarms, however conversely the system may increase collision risk in the case of malfunction ${ }^{[20]}$. In the next step, we would verify the influence of the false alarms and the missing alarms on the drivers' behavior.

Although the experimental results showed the statistically-significant effectiveness of the proposed FOCWS as mentioned above, there is undeniable sense that the number of participants was sixteen which was not enough to verify the effectiveness completely. Accordingly, we plan to perform additional experiments in the future. 


\section{References}

1. Takahashi, H.: 'Summary of the eighth fundamental Traffic Safety Program', Journal of the Society of Automotive Engineers of Japan, 2006, 60, (12), pp.4-9

2. '2011 CSR Report - Balancing human activity with protecting the environment is what we seek -’ (Fuji Heavy Industries, 2011)

3. 'SAFETY ACTIVITIES - NISSAN'S APPROACH TO SAFETY -' (Nissan Motor Company, August 2006)

4. Coelingh, E., Eidehall, A., Bengtsson, M.: 'Collision warning with full auto brake and pedestrian detection - A practical example of automatic emergency braking -', Proc. of 13th Int. IEEE Conf. on Intelligent Transportation Systems, Madeira Island, Portugal, September 2010, pp.155-160

5. Bogenrieder, R., Fehring, M., Bachmann, R.: 'PRE-SAFE in rear-end collision situations', Proc. of 21th Int. Technical Conf. on the Enhanced Safety of Vehicles, Stuttgart, Germany, June 2009, Paper No.09-0129

6. Ben-Yaacov, A., Maltz, M., Shinar, D.: 'Effects of an in-vehicle collision avoidance warning system on short- and long-term driving performance', Human Factors: The Journal of the Human Factors and Ergonomics Society, 2002, 44, (2), pp.335-342 
7. Jamson, A.H., Lai, F.C.H., Carsten, O.M.J.: 'Potential benefits of an adaptive forward collision warning system', Transportation Research Part C, 2008, 16, (4), pp.471-484

8. Chang, S.H., Lin, C.Y., Hsu, C.C., Fung, C.P., Jiun-Ren Hwang, J.R.: 'The effect of a collision warning system on the driving performance of young drivers at intersections':

Transportation Research Part F, 2009, 12, (5), pp.371-380

9. Adell, E. a, Várhelyi A., Della Fontana M.: 'The effects of a driver assistance system for safe speed and safe distance - A real-life field study -', Transportation Research Part C: Emerging Technologies, 2011, 19, (1), pp.145-155

10. Bella, F., Russo, R.: 'A Collision Warning System for rear-end collision: a driving simulator study', Procedia Social and Behavioral Sciences, 2011, 20, pp. 676-686

11. Hiraoka, T., Tanaka, M., Kumamoto, H., Izumi, T., Hatanaka, K.: 'Collision risk evaluation index based on deceleration for collision avoidance (first report) - Proposal of a new index to evaluate collision risk against forward obstacles -', Review of Automotive Engineering, 2009, 30, (4), pp.429-437.

12. Hiraoka, T., Tanaka, M., Takeuchi, S., Kumamoto, H., Izumi, T., Hatanaka, K.: 'Collision risk evaluation index based on deceleration for collision avoidance (second report) Forward obstacle collision warning system based on deceleration for collision avoidance -', Review of Automotive Engineering, 2009, 30, (4), pp.439-447. 
13. Hiraoka, T., Takada, S.: 'Collision risk evaluation based on deceleration for collision avoidance', Transactions of the Society of Instrument and Control Engineers, 2011, 47, (11), pp.534-540

14. Kitajima, S., Marumo, Y., Hiraoka, T., Itoh, M.: 'Comparison of evaluation indices concerning estimation of driver's risk perception - risk perception of rear-end collision to a preceding vehicle -', Review of Automotive Engineering, 2009, 30, (2), pp.191-198.

15. Fitts, P. M., Seeger, C, M.: 'S-R Compatibility - Spatial characteristics of stimulus and response codes -', Journal of Experimental Psychology, 1953, 46, pp.199-210

16. T. Hiraoka, K. Nakata, M. Tanaka, H. Kumamoto: 'Situation-adaptive warning timing of a forward obstacle collision warning system', Proc. of 15th World Congress on Intelligent Transport Systems, CD-ROM, 2008

17. Ishibashi, M., Okuwa, M., Akamatsu, M.: 'Development of driving style questionnaire and workload sensitivity questionnaire for drivers' characteristic identification', Proc. of 2002 JSAE Annual Congress, 2002, No.55-02, pp.9-12

18. Vassilis, C., Stylianos, P.: 'Human-machine collaboration through vehicle head up display Interface', Cognition, Technology \& Work, 2010, 12, (1), pp 41-50 
19. Park, S.H., Kim, K.: 'Driver-View based Augmented-Reality HUD System: Concept and Background', Proc. of 19th ITS World Congress, Vienna, Austria, October 2012, Paper No.AP-00162

20. Abe, G., Richardson, J.: 'The influence of alarm timing on driver response to collision warning systems following system failure', Behaviour \& Information Technology, 2006, 25, (5), pp. 443-452 


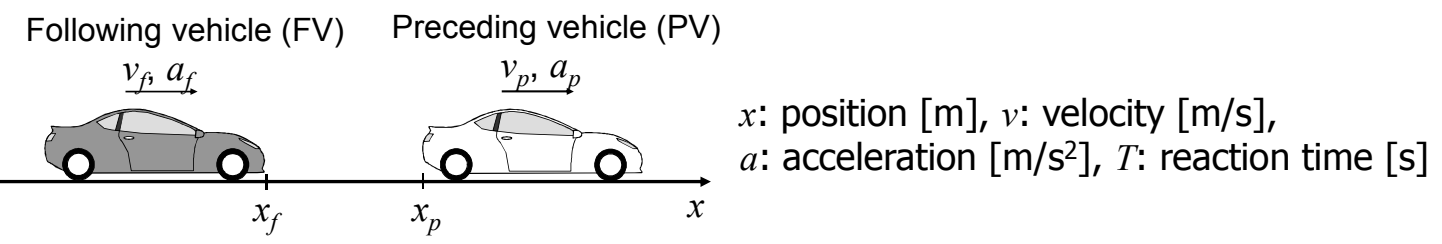

Fig. 1: Definition of variables 


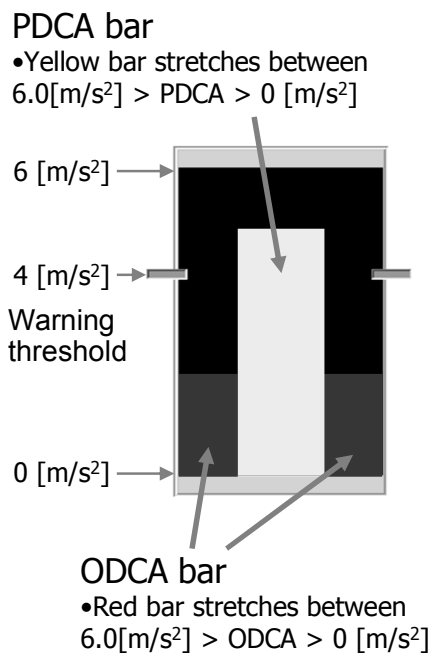

(a) DCA-FOCWS in the previous study

\section{PDCA frame}

-Yellow frame turns on

when PDCA $>4.0\left[\mathrm{~m} / \mathrm{s}^{2}\right]$

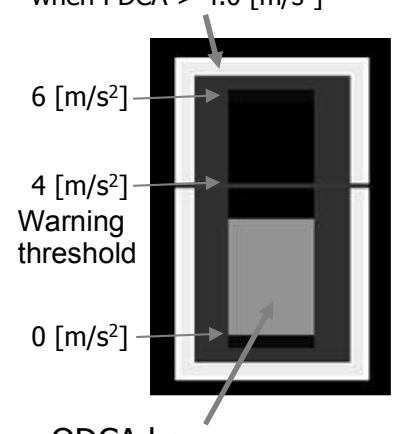

ODCA bar

-Orange: Driver's deceleration is

not enough to avoid the collision.

-Light blue: Driver's deceleration

is enough to avoid the collision.

(b) DCA-FOCWS

in the present study

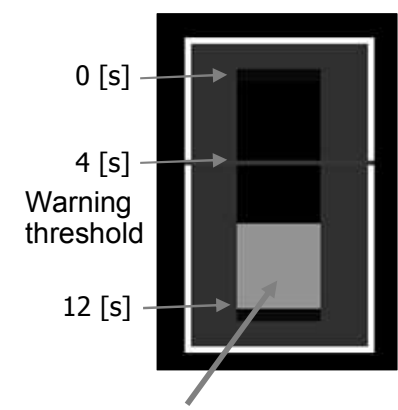

TTC bar

-Orange bar stretches

between $12[\mathrm{~s}]>\pi \mathrm{TC}>0[\mathrm{~s}]$

(c) TTC-FOCWS

in the present study

Fig. 2: Visual interfaces of FOCWS 


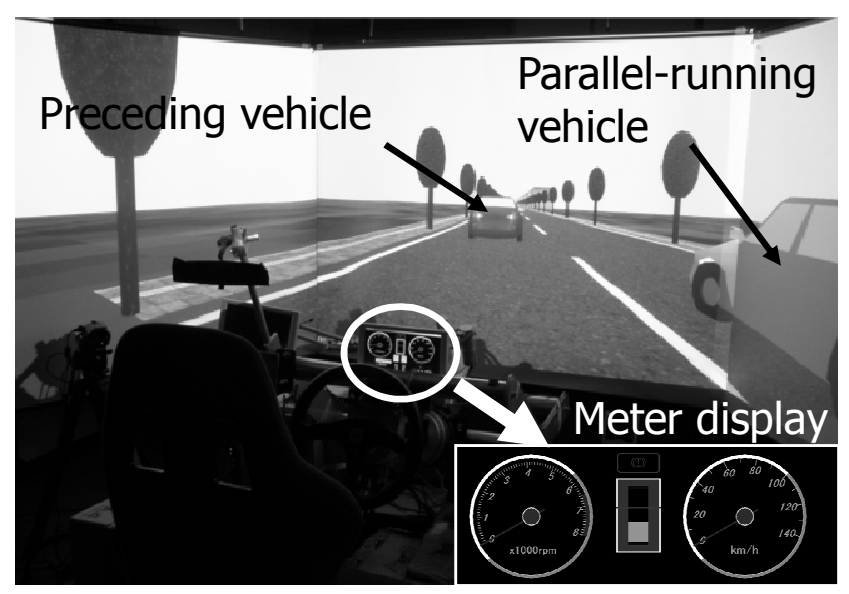

Fig. 3: Front view of driving simulator 

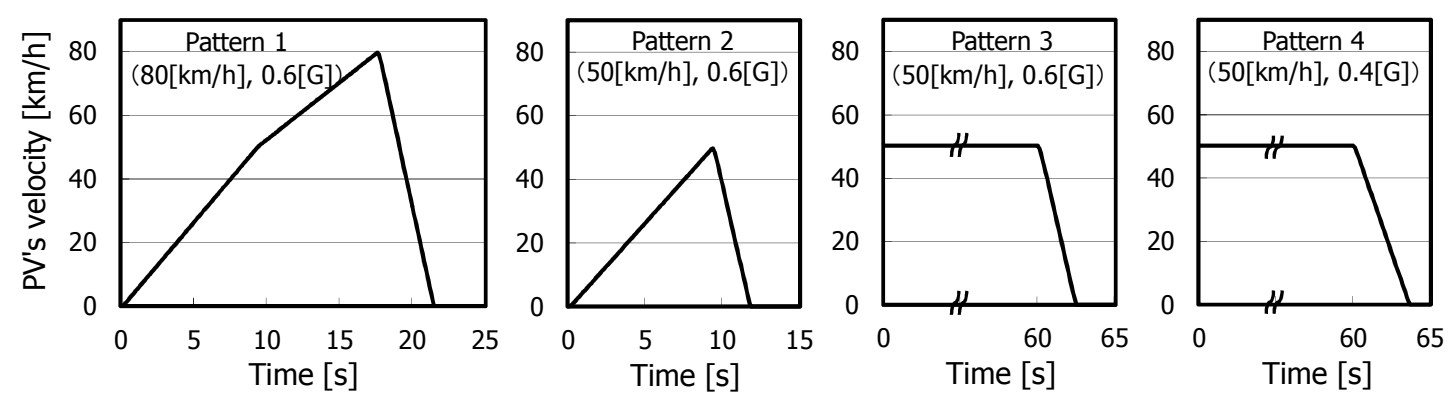

Fig. 4: Preceding vehicle deceleration patterns 

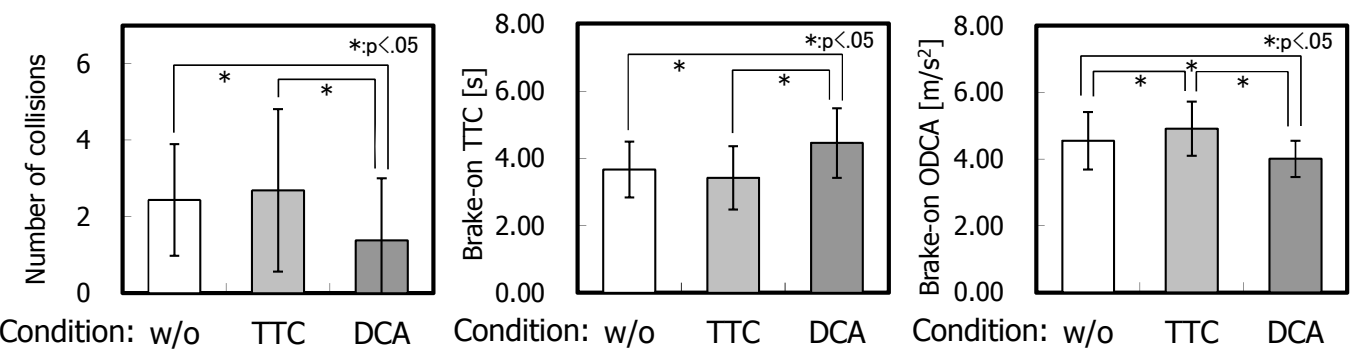

(a) Number of collisions

(b) Brake-on TTC

(c) Brake-on ODCA

Fig. 5: Experimental results 


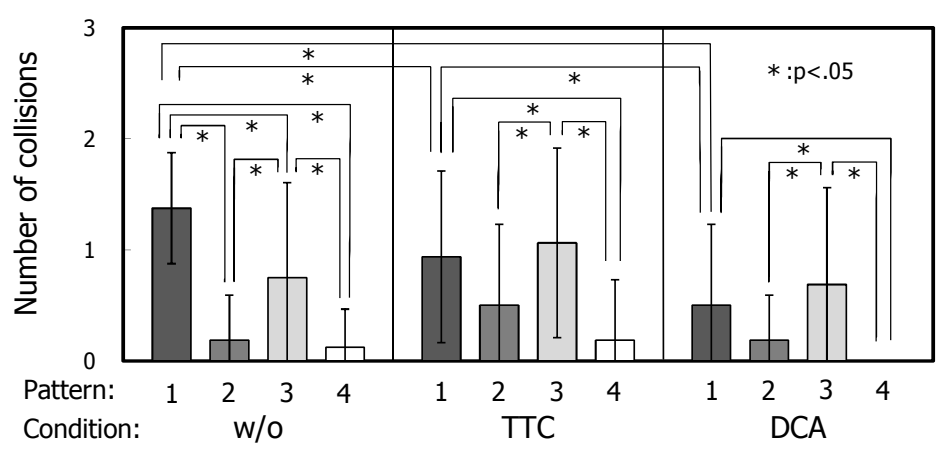

(a) Number of collisions

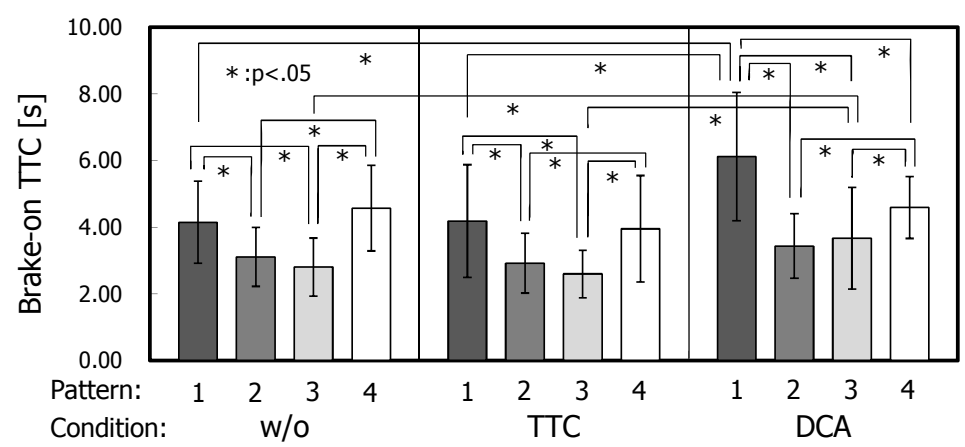

(b) Brake-on TTC [s]

Fig. 6: Experimental results by types of deceleration pattern 

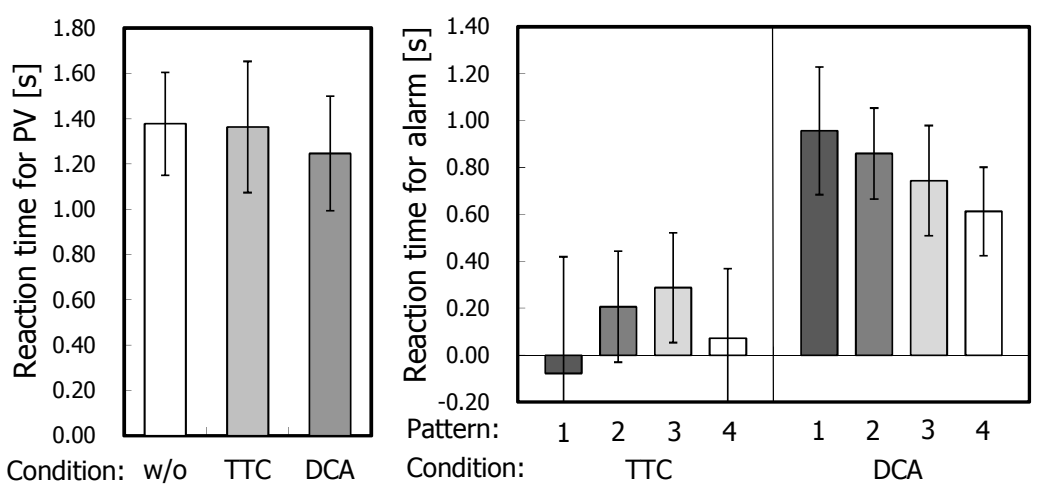

(a) Reaction time for preceding vehicle (b) Reaction time for alarm

Fig. 7: Reaction time 


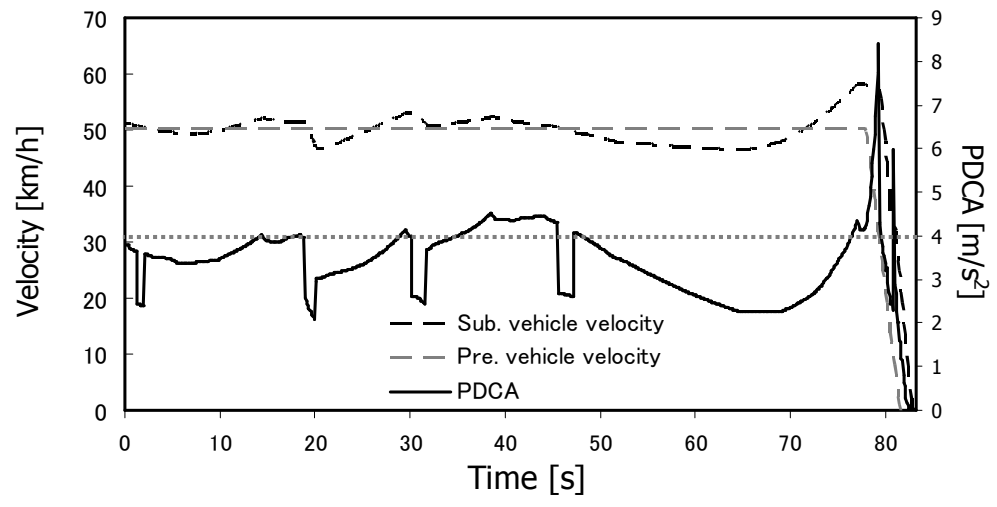

Fig. 8: Driving behavior of Sub. \#8 


\section{Table 1: Experimental results of four participants}

(a) Total time of PDCA $>4[\mathrm{~s}]$

\begin{tabular}{|c||c|c|c|}
\hline Condition & w/o & TTC & DCA \\
\hline \hline Sub. \#3 & 182.0 & 240.2 & 167.9 \\
\hline Sub. \#8 & 237.8 & 192.1 & 156.4 \\
\hline Sub. \#11 & 149.7 & 219.8 & 97.5 \\
\hline Sub. \#15 & 152.4 & 194.4 & 99.2 \\
\hline \hline Ave. & 180.5 & 211.6 & 130.3 \\
\hline
\end{tabular}

(b) Number of collisions

\begin{tabular}{|c||c|c|c|}
\hline Condition & w/o & TTC & DCA \\
\hline \hline Sub. \#3 & 3 & 0 & 0 \\
\hline Sub. \#8 & 5 & 5 & 1 \\
\hline Sub. \#11 & 1 & 1 & 0 \\
\hline Sub. \#15 & 1 & 2 & 0 \\
\hline \hline Ave. & 2.50 & 2.00 & 0.25 \\
\hline
\end{tabular}



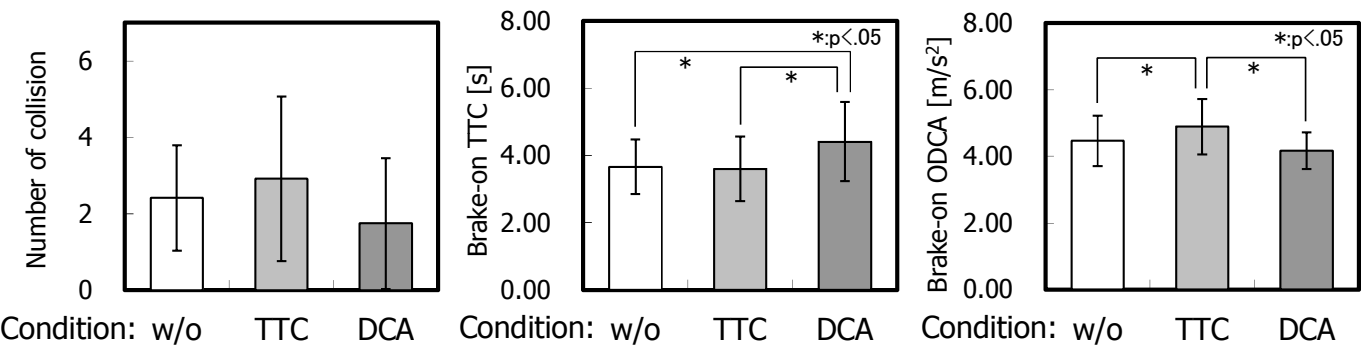

(a) Number of collisions

(b) Brake-on TTC

(c) Brake-on ODCA

Fig. 9: Experimental results of 12 participants 

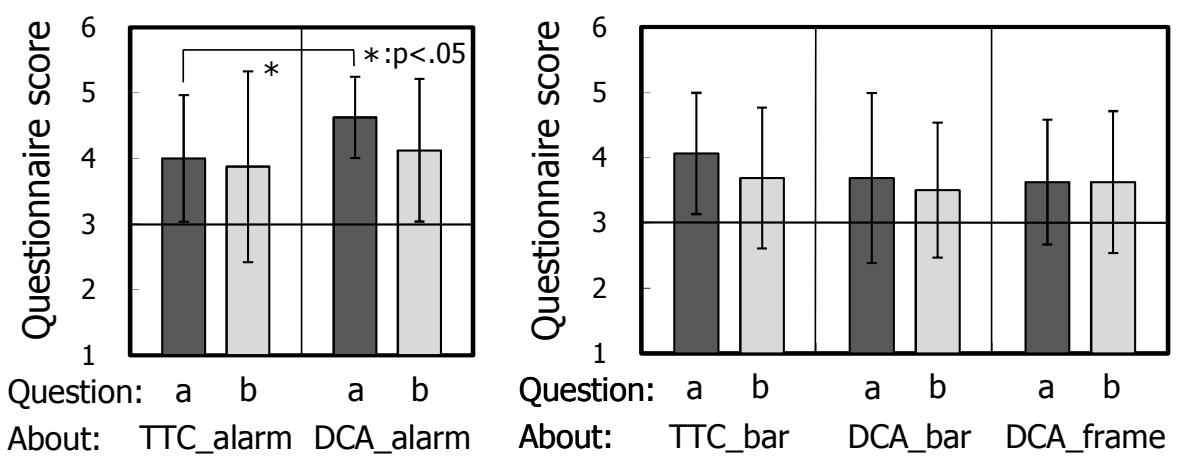

(a) Questions 1-a and 1-b

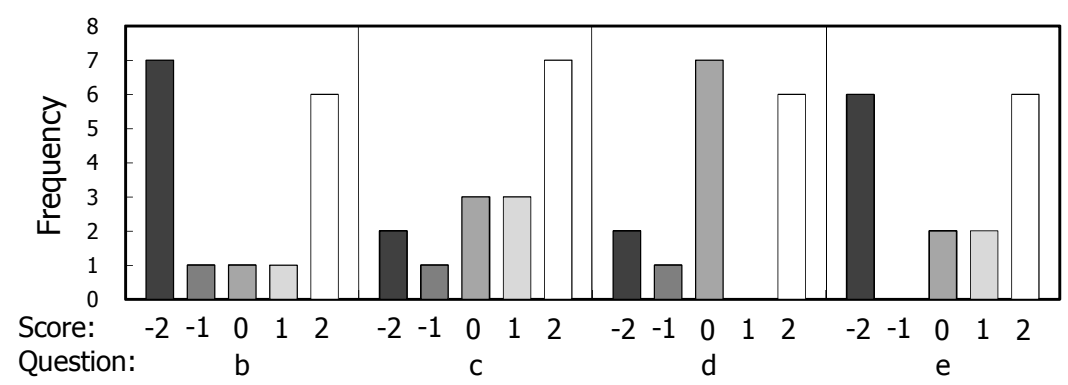

(b) Questions 2-b 2-e

Fig. 10: Questionnaire scores 


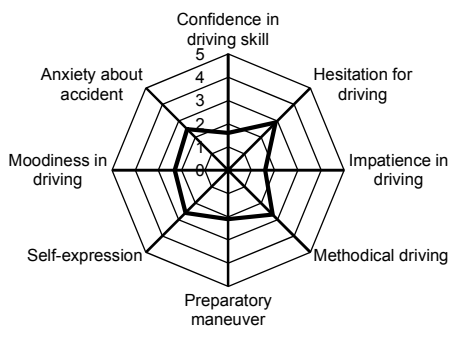

(a) Group 1

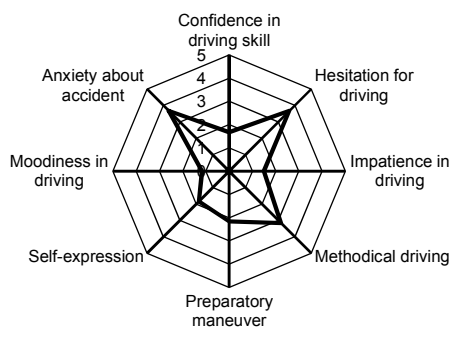

(b) Group 2

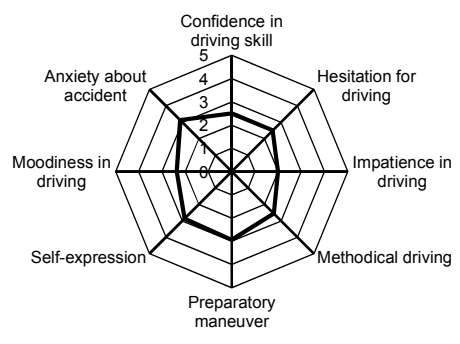

(c) Group 3

Fig. 11: DSQ score 

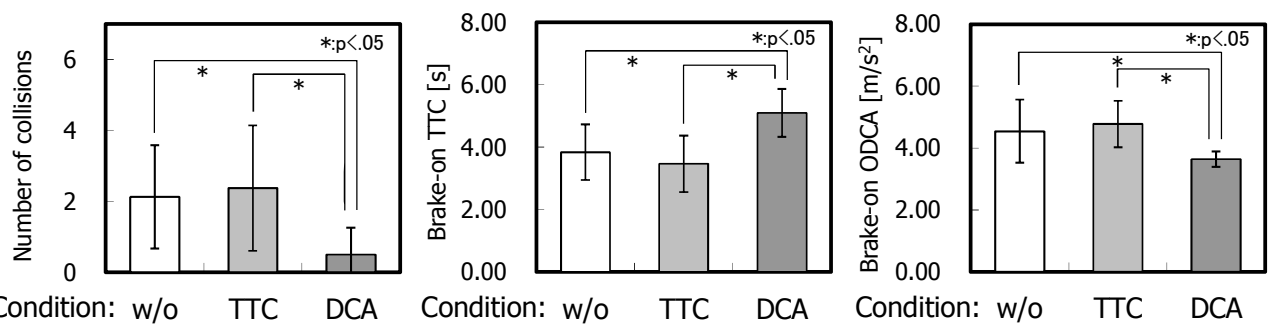

(a) Group A
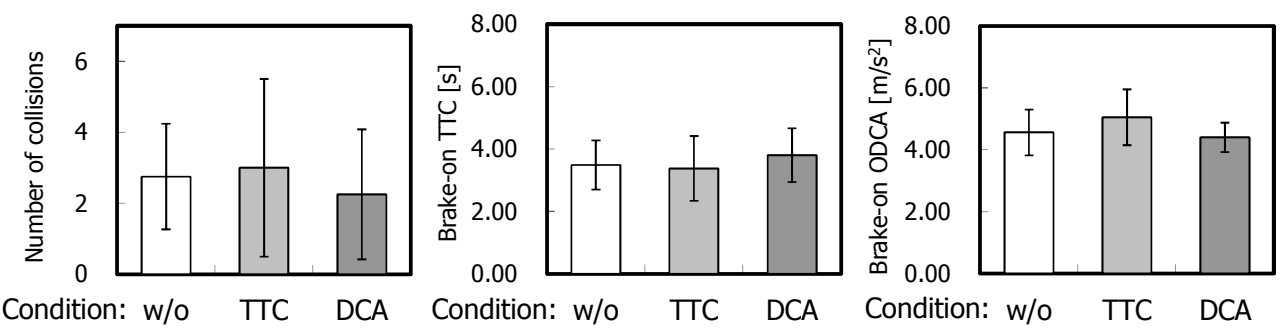

(b) Group B

Fig. 12: Performance differences between Group A and Group B 